\title{
Towards a Generalized System Dynamics Model for Product Design \& Adoption
}

\author{
Marc-Andre CHAVY-MACDONALD ${ }^{1}$, Kazuya OIZUMI and Kazuhiro AOYAMA \\ Department of Systems Innovation, School of Engineering, University of Tokyo, Japan
}

\begin{abstract}
Product Design is at the core of engineering, and is often transdisciplinary. Holistic product models and viewpoints are needed for making early design decisions. Such models must place the product in its relevant, dynamic environment, thus taking into account societal factors in addition to engineering disciplines. System Dynamics (SD) can apprehend semi-qualitative societal variables. It also has the advantage of transparency, and demonstrates the essential qualitative dynamics of large complex systems. Several SD model types and fragments ("molecules") have been successfully used around product development, but their integration is unclear. We integrate several SD molecules to form a simple, yet holistic model for product design. It is based on the Bass diffusion model; the main novelty is combining this with customer preferences. External-facing product functions contribute to utility, backed by internal functions. It proposes an expression for part-utilities, and distinguishes between types of network effects. The model structure is generalizable to products, services, and wider ecosystems. It systematizes data acquisition by showing gaps for input by e.g. conjoint analysis, and allows value-of-information calculations. We demonstrate the model structure using the introduction of the household freezer. Product success is dominated by successive SD regimes: initially technological improvement, modulated by a niche and durable goods replacement rate, then by network effects. The next step is better quantifying the more difficult factors, giving standardized ranges of values \& functions, based on compiled empirical data across a range of products. This research furthers SD "molecules", aiming to clarify the "basic physics" of product design \& adoption.
\end{abstract}

Keywords. Product Design, System Dynamics, Systems Engineering, Sociotechnical System, Product Diffusion

\section{Introduction}

Product Design is a core function of engineering, and is necessarily transdisciplinary. Holistic product models are needed to build a common understanding \& support decisions across engineering disciplines, but also with business functions such as marketing \& strategy to ensure market acceptance. This need is growing due to the increasing pace of societal change, making synchronous understanding \& speed necessary in product development. Within engineering, paradigms such as ModelBased Systems Engineering (MBSE) \& Product Lifecycle Management (PLM) seek to create integrated or connected models, yet these are generally separate from business modeling. For engineering models to bridge the discipline gap with business, one necessary characteristic is they must apprehend change, yet they are typically static.

\footnotetext{
${ }^{1}$ Corresponding Author, Mail: marc@m.sys.t.u-tokyo.ac.jp.
} 
Similarly marketing variables and product diffusion are often driven by soft factors, thus a holistic product design \& diffusion model must integrate soft variables. Finally, to effectively impact practice, a model must be clear \& easily understandable to all.

A transdisciplinary approach to modeling is needed. One modeling paradigm that is discipline-agnostic and has the needed characteristics - dynamic, incorporating soft variables, and emphasis on clarity - is System Dynamics. The purpose of this research is to test the suitability of System Dynamics as a framework for a generalizable, holistic product design \& diffusion model.

\section{Research Question}

Can a System Dynamics model capture the dynamics of (new) product design(s) and acceptance? Is the model useful, insightful, simple? How to build it, in general?

Thus as key contribution, this paper seeks to generate an integrated System Dynamics (SD) structure that can advantageously capture many dynamics of product design \& acceptance, in a general way. To do so, the steps of the research are (Fig. 1):

1. Identify the phenomena to capture, using engineering \& economic/business theory, and an Innovation Studies narrative; also specify criteria to evaluate whether they have been adequately captured;

2. Build the SD model: from features of the product \& market, identify relevant SD building blocks, integrate them. A primary contribution is the identified building blocks, mapping to key features, \& their integration;

3. Evaluate the SD model according to the established criteria, such as model coverage $\&$ ease of use.

\section{Organisation \& contribution of this paper}

This paper is organized as follows. Section 1 introduces the different literatures, from several disciplines, relevant to product design \& development, and diffusion \& acceptance. Section 2 describes the research method: formulating a research hypothesis, gathering data, building a System Dynamics model, simulating it, and verifying the hypothesis. Section 3 describes the main results and research contribution: an initial generalized SD model structure for product design \& diffusion, consisting of several molecules and able to apprehend many key features of product development \& diffusion. Finally, Section 4 discusses the model's results and performance vs. criteria.

\section{Literature}

There are several different perspectives and literatures on product design \& diffusion, both within and beyond System Dynamics modeling. We briefly introduce them here.

\subsection{Holistic, transdisciplinary models of product design \& diffusion}

There are many models of new product $\&$ innovation diffusion [1] and of product acceptance more generally, e.g. in marketing [2], [3], though diffusion studies span many other fields like innovation studies \& sociology. In marketing, the introduction of the Bass model has led to extensive research [4], [3]. Dynamic diffusion models, with 
market saturation changing over time, date from the 1970s, while attribute-based flexible models (which depend on product characteristics) exist since the 1980s. Though they are used normatively for marketing product planning, these models do not consider the engineering perspective, of a product composed of functions \& components - with constraints. There is also room for research on integrating technological progress, and marketing attributes with the Bass model [3].

On the other hand, the field of Systems Engineering \& Product Design proffers many holistic product methodologies \& models for early decisions, e.g. SysML (System Modeling Language) (reviewed by e.g. [5]), but these rarely consider the business side \& product diffusion. Marketing and engineering design are considered quite different perspectives of products (Table 1 of [2]). Indeed, there are research opportunities to link models of consumer preferences with engineering models describing technical constraints [2]. QFD is one of the rare paradigms that bridges this gap, but is static [5].

\subsection{System Dynamics models for product design}

System Dynamics (SD) modeling is commonly used in business \& policy [6], with the main advantages of (a) visually showing dynamic structure of a problem, and (b) apprehending social, soft variables as well as technical, hard ones. The thesis of SD is that feedbacks, delays, stocks \& flows can simply, endogenously capture most complex behaviours at large scales. Originating with Jay Forrester in the 50s \& 60s, it was made famous by the Club of Rome's Limits to Growth. More recent champions include Peter Senge [7], who also popularized the idea of system archetypes, similar to molecules these are simple model structures found in many applications, pointing to common system structures, such as delayed negative consequences to simple fixes [8], [9].

Many SD models (\& molecules) related to product development \& diffusion exist, though fewer for design. [6], a standard text, presents SD models of product diffusion, durable goods replacement, network effects, and the many positive feedbacks of corporate growth - and many others. [10] provide a SD model incorporating conjoint analysis for use in automotive industry strategy, emphasizing embedding customer preferences in the model. [11] deploys an innovation diffusion SD model with successive product generations for Nike. [12] gives 5 SD models for corporate strategy, including technology substitution and the stock-flow structure of R\&D management. [13] introduces the idea of utilizing the functional architecture of a system as basis for an SD model. [14] relates design parameters to utility and alternative fuel vehicle adoption via SD. [15] models a double market incorporating a diffusion model, conjoint analysis \& network effects, utilizing additive logarithmic utility functions. Very few SD models incorporate engineering design parameters, and if so do not link to utility, product diffusion $\&$ hence profitability.

\section{Research Method}

Though there are many SD models around product diffusion, there is a need to connect these with product design decisions, particularly showing the typical model structures (molecules) needed and hence a generalized model for use by product designers \& planners. Thus we seek to create a novel SD model structure, following the process in Figure 1, described in detail in the following section. 


\subsection{Formulate hypothesis \& criteria}

Following a simple literature review, a hypothesis was formulated from the research question as follows:

A Bass model-based System Dynamics model of a few molecules can simply capture most behaviour of product diffusion, and relate to design parameters, enabling holistic design. Evaluation of this hypothesis will necessarily be qualitative $\&$ approximate, yet we can set criteria. Inspired by [16] \& [17], we choose ease of model creation \& calibration, usable in collaborative (transdisciplinary) design, proven applicability in industry, and model coverage of essential phenomena.

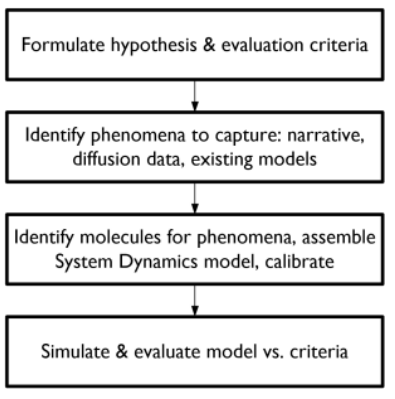

Figure 1. Research method.

\subsection{Gather data}

As a starting point, we rely on a case study: the introduction of the household freezer in the UK from the 1960s to 1990 s, as documented by [18]. This well-known research provides a holistic, convincing narrative of the phased introduction of a novel product in society, from the perspective of Domestication Theory. Many causal factors for product diffusion are explored over time, be they technological, design, economic, social, or other related markets. Its holistic approach makes it a good candidate for identifying the various phenomena that need to be captured in a model of product design $\&$ diffusion. In addition to factors identified by the narrative, it refers to data on market penetration [19].

In addition, survey of the SD literature revealed other important factors for diffusion that probably need to be captured. Existing modeling work leaves us molecules of diffusion phenomena, often with some numerical calibration [6], [8]. This data provides us with a "bottoms-up" complement to the "top-down" modeling needs from [18].

\subsection{Model-building process}
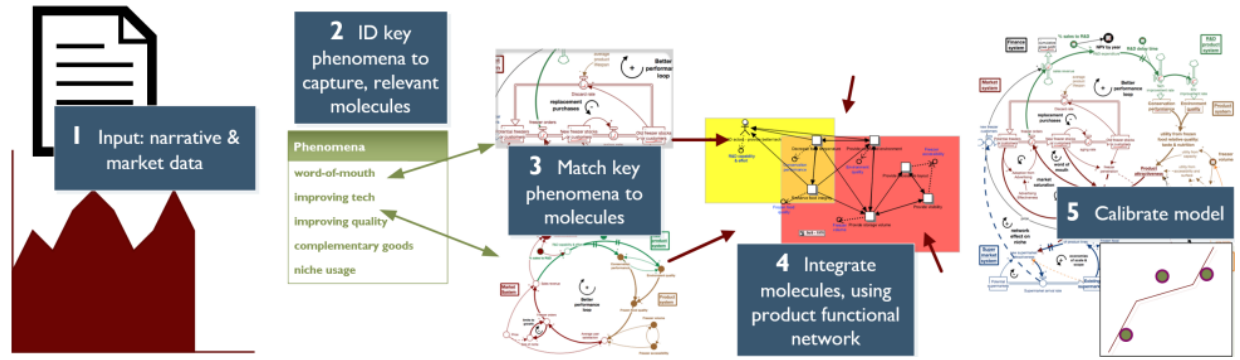

Figure 2. Model-building process, in 5 steps. For more detail of functional network, see [13], [20].

Figure 2 depicts the SD model-building process as done here. From a narrative \& market data, key phenomena to capture in the model are identified. Similarly, likely SD model molecules are listed, and there is a matching process: key phenomena are associated to molecules. Molecules are selected for inclusion based on their utility in explaining the phenomena. The fourth step is to integrate the molecules, also using a 
network of the essential functions of the product (Functional Dependency Network, FDN - for details see [13] or [22]. The main product functions are either contributing to customer value (and thus related to adoption), or are internal, and support customerfacing functions (and often affected by technological change) [21]. The functions' performance level is related to design parameter choices. Finally once the model is integrated, numerical calibration is done - in this, established parameter values for model molecules are used as much as possibly, to simplify.

\subsection{Simulate \& verify hypothesis}

The final phase of the research is to simulate the model, for a variety of test cases, and observe how well the essential phenomena are captured. Can the model simply explain a variety of cases, by tuning only a few parameters?

Finally the model coverage is rated on a 3-point scale: Basic, Moderate, Strong [16]. So are the ease of model creation, its understandability to a transdisciplinary team, and applicability of the process in industry.

\section{Results}

\subsection{Key phenomena for freezer design \& diffusion, and mapping}

Some key phenomena in freezer diffusion identified from [18] are shown in the first two columns of Table 1 . They are divided into 2 time periods, corresponding to the "introduction" and "establishment" of the product. [18] is a rich narrative and this table is only summarizing a few of its essential points. Note the substantial qualitative shift in narrative between the periods, needing different models.

The third column of Table 1 shows System Dynamics molecules which can capture the identified phenomenon. All are pre-existing, save the relation to the FDN. Other SD molecules might also fit these phenomena, though the best-known were chosen. In addition, other phenomena and causal factors were described in the narrative (notably changing social roles), but these were selected as some of the most essential, and susceptible to modeling. Thus this mapping is not unique, but a starting point.

Table 1. Example key identified phenomena from innovation studies narrative, \& associated SD molecules.

\begin{tabular}{ccc}
\hline Period & Identified phenomena & System Dynamics molecules \\
\hline 60s \& early 70s & Adoption by niche population & Bass model for niche market \\
"introduction" & Poor but rapidly improving tech & R\&D cycle \\
& Isolated product, unconstrained size & Purt-utilities (\& FDN) \\
& Utility driven by freezing food quality & Aging chain \\
& Part-utilities \\
\hline Late 70s \& 80s & Becomes mass market via supermarkets & Bass model, network effect on niche \\
"establishment" & Supermarkets, fitted kitchen increase utility & Network effects \\
& Physically constrained design, for kitchen & Part-utilities (\& FDN) \\
& Utility driven by new use case, whole system & Part-utilities, all network effects \\
\hline
\end{tabular}

\subsection{SD molecules selected}

Figure 3 shows a SD molecule of Bass diffusion. The Bass model is often used to model innovation diffusion, generating an S-curve similar to the logistic model and epidemics [6], [4], [11]. System Dynamics exposes its structure: potential customers 
and adopters are stocks, with sales (adoption) a flow. Adoption stems primarily from word-of-mouth: product "attractivity" is constant, and sales proportional to contact between adopters and potentials. So there is a positive feedback loop at early times, with market saturation later as potential customers decrease. Finally, advertising (or e.g. direct sales) starts the model from 0 adopters.

Figure 4 shows an SD molecule of an aging chain, extending the Bass model for durable goods and repeat customers [6], [10]. Freezers age and are eventually discarded, after which customers must again go through the word-of-mouth cycle. Replacement purchases act as a stabilizing feedback on sales.

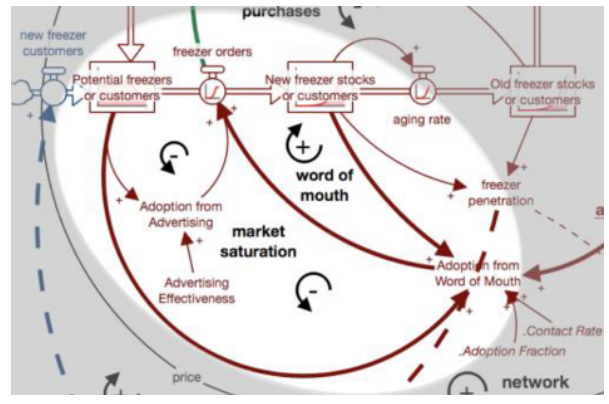

Figure 3. Bass diffusion of products by word-ofmouth.

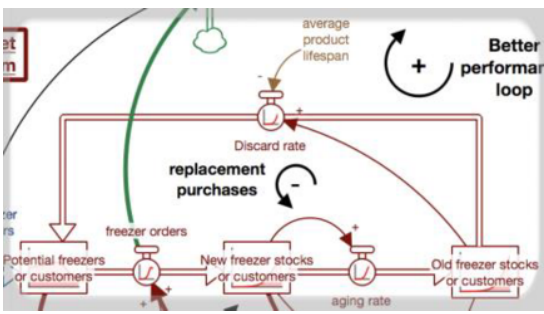

Figure 4. An aging chain, of consecutive stocks. New freezers become old over time, and are eventually discarded, creating new potential customers. They must re-adopt the freezer.

Figure 5 shows the R\&D or product development cycle SD molecule, a key driver of corporate growth. It is a positive feedback loop, as sales revenue leads to improved products via $R \& D$ or design activities, in turn increasing sales [12], [6]. R\&D (or indeed design) capability can be represented as a stock-flow, delayed structure. In this case, technology contributes to customer-facing functions, and product attractiveness.

In the SD molecule of Figure 6, product attractivity is not constant, but decomposed into contributing part-utilities, which themselves can be affected by other factors, and dynamic [10], [14], [15]. The functional form of part-utility functions is debated, with arguments for both additive $\&$ multiplicative. We use additive logarithms, allowing both utility addition and modulation - and ever-increasing attractivity from improved features, even if insignificant.

Figure 7 shows a SD molecule for network effects between 2 quantities [12], [6]. Network effects are important positive feedbacks for complementary goods and when attractiveness depends on the user base; here the existing stock of supermarkets increases fitted kitchen install rate, and vice-versa. Network effects can also be

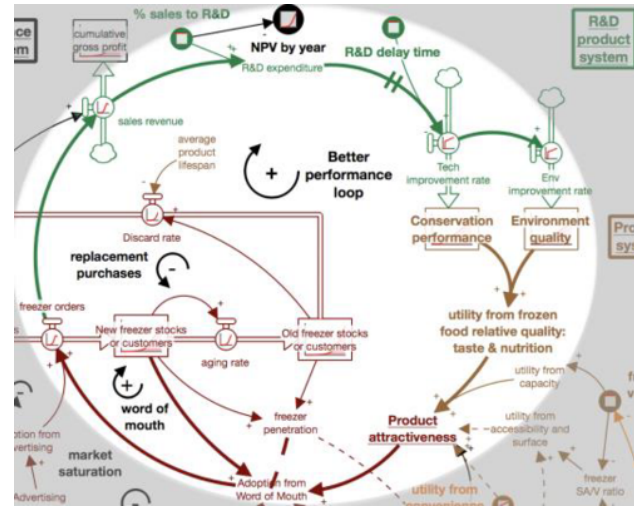

Figure 5. The R\&D cycle: revenue brings better products.

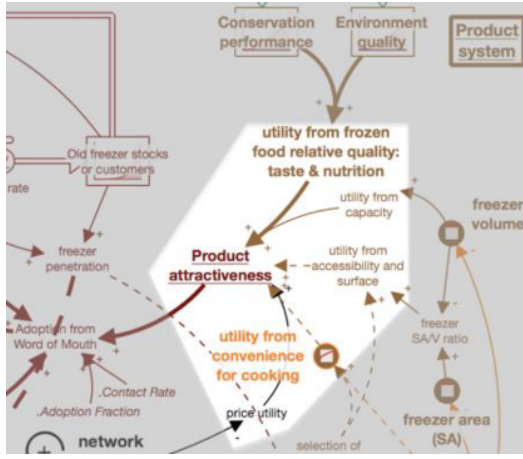

Figure 6. Part-utilities decompose product attractiveness, and can be related to a specific product feature. Here modeled as logarithms. 
modeled as impacting the potential customer pool - increasing the market niche.

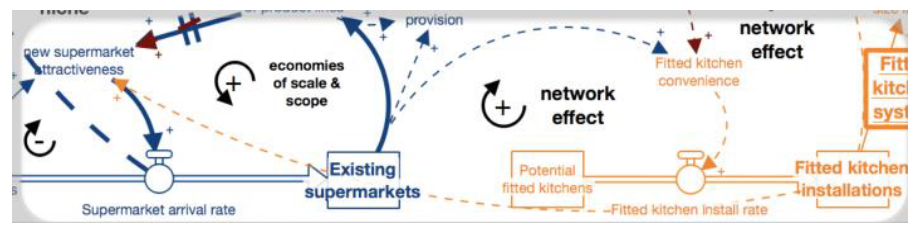

Figure 7. Example network effects, modeled as stocks increasing each others' flow rate (adoption).

\subsection{Molecule integration \& full model}

Figure 8 shows the whole model (simplified view). It is the basic structure capturing features from Section 3.1. The molecules from Figs. 3-7 are all integrated. The 6 systems are colour-coded: product, market, R\&D, finance and 2 complementary goods: supermarkets \& fitted kitchens. To try to improve clarity arrows are drawn as, in order of priority: thick if they belong to a feedback loop (except network effects), dotted if contributing to network effects, and both for the network effect on niche size.

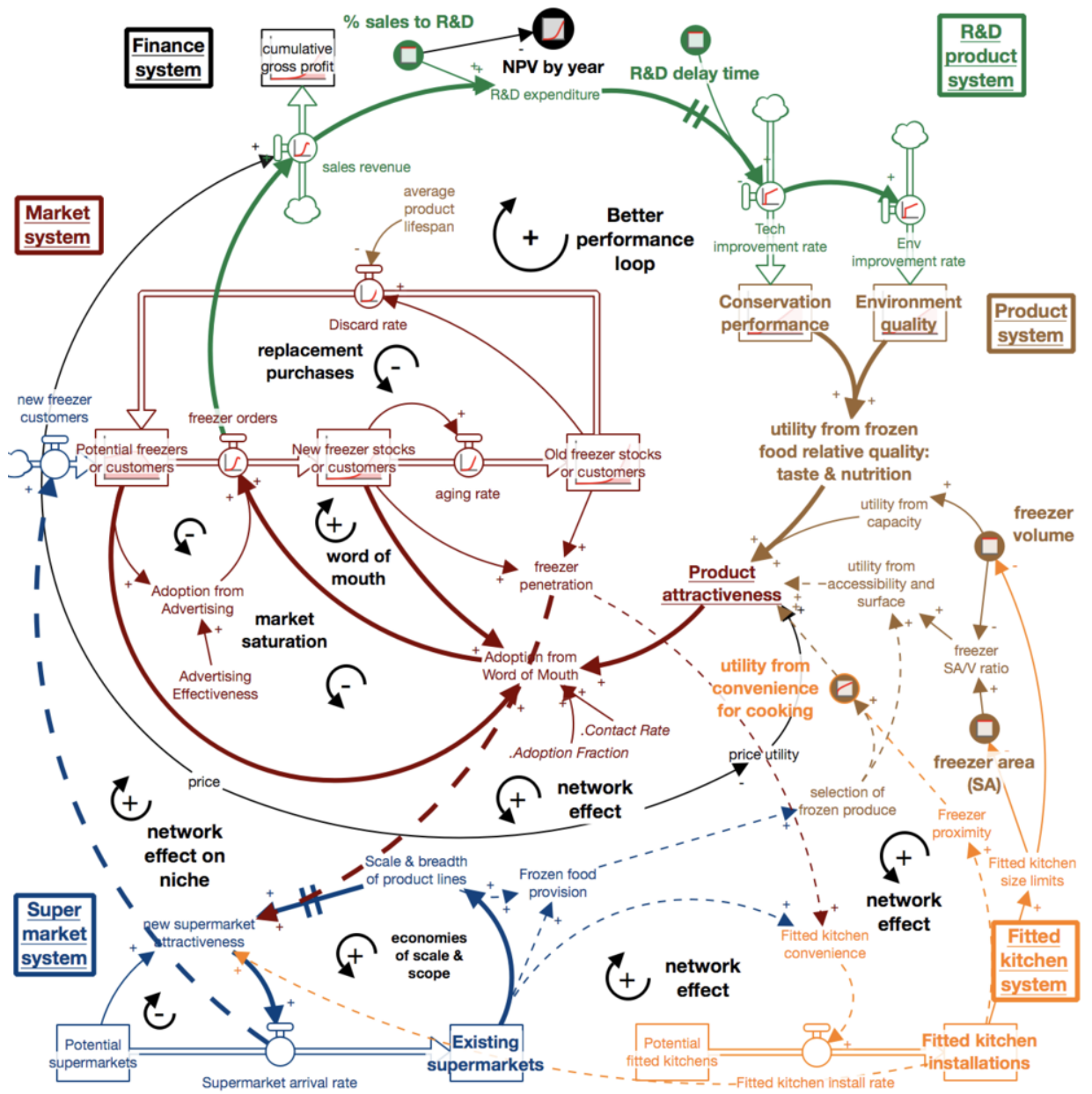

Figure 8. Full model of the integrated 5 molecules, and 6 systems. Note the dominant positive feedbacks. 
The dominant behaviour is of the Bass model, extended by repeat purchases and modulated by variable attractiveness, which is affected both by ongoing $R \& D$, various design choices, and importantly network effects. The main novelty is in integrating the Bass model with customer preferences (via attractivity), which can be linked both to part-utilities of customer-facing product functions (and product design), and internal technological functions affected by $\mathrm{R} \& \mathrm{D}$. Other functions (here utility from convenience for cooking) form the "gateway" for utility from complementary goods to improve product attractiveness: suitability for use with complementary goods then becomes a major purchasing criteria. Complementary goods can also have a different form of network effect, instead increasing the size of the market niche - this is shown by the thick dotted arrow, from supermarkets to new freezer customer arrival rate.

We note that the model as a whole is dominated by positive feedback loops, notably 4 network effects. The only negative feedbacks arise from replacement purchases and market niche saturation.

\subsection{SD simulation \& calibration}

Figure $9 \& 10$ show the simulation of the calibrated model for 2 cases: "introduction", and "establishment" (Table 1). Only 8 datapoints are shown, but there are many other constraints: introduction dates of freezers \& other products, supermarket etc. diffusion time, product lifespan, relative preference data, approximate niche size, and narrative on key factors [18], [19]. Also, many parameters of the molecules have guidelines for typical values, e.g. advertising and R\&D effectiveness. Three fitting parameters are used for the chest case: product attractiveness and size of niche, and (constrained) R\&D effectiveness. Only one additional parameter - network effect impact on attractiveness - is used to fit the remaining 4 points, once the rest of the model is "activated".

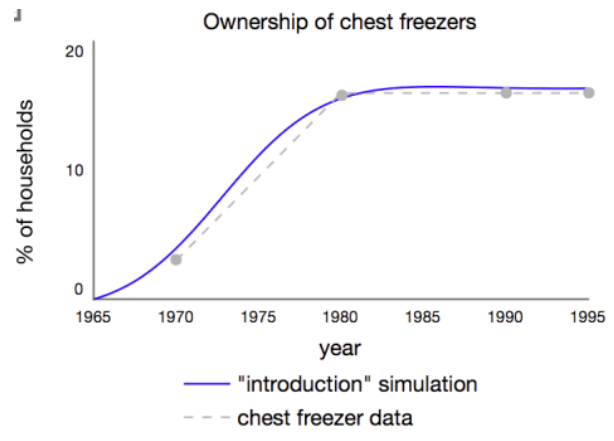

Figure 9. Model fit for "introduction" case.

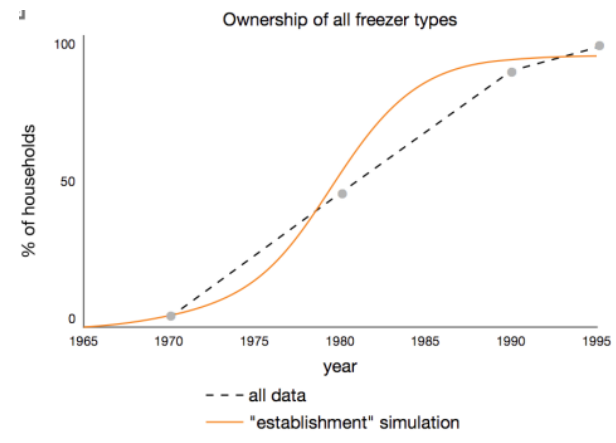

Figure 10. Model fit for all freezers.

Several regimes are apparent: in Figure 9, an initial low attractiveness is quickly improved by technology, leading to the chest freezer niche being largely filled by 1980 . Figure 10 shows the effect of supermarket \& fitted kitchen gradual arrival from 1970.

Because the diffusion of the later fridge-freezer (a hallmark of fitted kitchens, in the "establishment" era) occurred at a slower rate than chest freezers, we conclude that product attractiveness was not much higher. Thus, the network effects on product attractiveness are unlikely to be dominant. Rather, the expansion of the market niche, by the population's gradual acceptance of cars \& supermarkets, was likely a key factor. 


\section{Discussion}

Despite the paucity of data, this structural model's ability to capture diffusion phenomena is good. It is readily interpretable and can thus offer physics-based insights. Proper calibration would include e.g. conjoint analysis to determine part-utilities. The structure provides a framework for data, and can be used to determine value of information, by computing sensitivity of NPV to parameters (e.g. preferences).

\subsection{Limitations}

The model is not nearly fully validated - but the direction and early results appear promising. Many important factors are excluded, notably the impact of competitor action, standards wars, and the link of design attributes to price.

It is also likely different narratives of product will reveal different key phenomena and causalities, and hence a different model will result. We make no claim to universality, but rather to some amount of generality - the phenomena of Table 1 are doubtless common in product diffusion, and thus widely applicable.

\subsection{Evaluating the model \& hypothesis}

Table 2. Evaluating the model using the criteria (Basic, Moderate, Strong). Centre column adapted from [17].

\begin{tabular}{ccc}
\hline Criteria & System Dynamics & Present model \\
\hline Ease of model creation \& calibration & $\mathrm{B}$ & $\mathrm{M}$ \\
Directly usable in collaborative (transdisciplinary) design & $\mathrm{M}$ & $\mathrm{S}$ \\
Proven applicability in industry & $\mathrm{S}$ & $\mathrm{M}$ \\
Model coverage of essential phenomena & - & $\mathrm{S}$ \\
\hline
\end{tabular}

Table 2 shows the evaluation of the model according to the 4 established criteria. Using the pre-existing evaluations in [16] \& [17] as a guide, we first rate System Dynamics as a global methodology. System Dynamics models are considered difficult to calibrate, warranting a "Basic", but are fair as a collaborative tool, and have a history of usage in industry. The present model type is somewhat easier to create \& calibrate, coming from pre-existing (and somewhat calibrated) molecules with the basic structure provided. The model is designed for collaborative understanding by engineers \& marketers, so warrants a "strong", but this specific form is not proven to be practical. Its potential (as SD) warrants a "moderate", while the model capture of essential phenomena is very good. We emphasize the above is all tentative, an early indicator.

\section{Conclusion}

This paper has tried to clarify the "basic physics" of holistic, transdisciplinary product design $\&$ acceptance. It has done so by choosing System Dynamics molecules suited to capture the phenomena described in research on product diffusion. Integrating the molecules has led to guidelines and a generalizable model structure for deploying SD. The key message \& contribution is, this integrated SD structure can advantageously capture many of the dynamics of product design \& acceptance, in a general way.

The impact on the practitioner and field should be positive. Hopefully designers from a wide variety of industries might try \& use this model structure, and have another tool for reasoning about product design - using an established paradigm, SD. 
For SD researchers, we hope for renewed attention on the benefits of molecules [8], [9]. Design \& Systems Engineering researchers might also be attracted to the SD field.

\subsection{Further work}

Further validation work is needed: of this model with more data, of other narratives \& models, and of more standardized molecules. Eventually, we might hope for standard, validated modeling libraries for product design $\&$ acceptance, akin to engineering parts.

\section{References}

[1] E. Rogers, Diffusion of Innovations, 5th ed, The Free Press, New York, 2003.

[2] V. Krishnan and K. T. Ulrich, Product Development Decisions: A Review of the Literature, Management Science, 2001, Vol. 47, pp. 1-21.

[3] V. Mahajan, E. Muller, and F.M. Bass, New Product Diffusion Models in Marketing: A Review and Directions for Research, Journal of Marketing, 1990, Vol. 54, pp. 1-26.

[4] F.M. Bass, A new product growth model for consumer durables, Management Science, 1969, Vol. 15, pp. $215-227$.

[5] T. Tomiyama, P. Gu, Y. Jin, D. Lutters, Ch. Kind, and F. Kimura, Design methodologies: Industrial and educational applications, CIRP Annals, 2009, Vol. 58, pp. 543-565.

[6] J. Sterman, Business Dynamics: Systems Thinking and Modeling for a Complex World, McGraw-Hill, Boston, 2000.

[7] P.M. Senge, The Fifth Discipline: The Art \& Practice of The Learning Organization, Revised and updated, Currency Doubleday, New York, 2006.

[8] J. Hines, Molecules of Structure - Building Blocks for System Dynamics Models, Sloan working paper Version 2.02, MIT, Cambridge, MA, USA, 2005.

[9] S. ElSawah, A. McLucas, and M. Ryan, Generic and reusable structure in systems dynamics modelling: roadmap of literature and future research questions, Proceedings of the 2015 International System Dynamics Conference, Boston, 2015, accessed July 8 2019, https://www.systemdynamics.org/assets/conferences/2015/proceed/papers/P1078.pdf,.

[10] M. J. Schmidt and M. S. Gary, Combining system dynamics and conjoint analysis for strategic decision making with an automotive high-tech SME, Syst. Dyn. Rev., 2002, Vol. 18, pp. 359-379.

[11] V. B. Kreng and B. J. Wang, An innovation diffusion of successive generations by system dynamics An empirical study of Nike Golf Company, Techn. Forec. \& Social Change, pp. 77-87, vol. 80, 2013

[12] H.B. Weil, Application of system dynamics to corporate strategy: an evolution of issues and frameworks, Syst. Dyn. Rev., 2007, Vol. 23, pp. 137-156.

[13] S. Lee, W. Han, and Y. Park, Measuring the functional dynamics of product-service system: A system dynamics approach, Computers \& Industrial Engineering, 2015, Vol. 80, pp. 159-170.

[14] A. Neerkaje, Strategies for the introduction of alternative fuel vehicles in India, Master's thesis, Massachusetts Institute of Technology, 2013.

[15] J. Wang, J.-Y. Lai, and C.-H. Chang, Modeling and analysis for mobile application services: The perspective of mobile network operators, Techn. Forecasting and Social Change, 2016, pp. 146-163.

[16] M.-A. Cardin, Enabling Flexibility in Engineering Systems: A Taxonomy of Procedures and a Design Framework, J. Mech. Des, 2013, Vol. 136, 011005.

[17] M.-A. Chavy-Macdonald, K. Oizumi, Y. Kishita, and K. Aoyama, A System Dynamics and scenarios framework for determining dynamic product architecture goals in a System-of-Systems, IEEE Systems Journal, 2018, Vol. 13, Issue 2, pp. 1957 - 1968.

[18] E. Shove and D. Southerton, Defrosting the Freezer: From Novelty to Convenience A Narrative of Normalization, Journal of Material Culture, 2000, Vol. 5, pp. 301-319.

[19] B. Boardman, K. Lane, M. Hinnells, N. Banks, G. Milne, A. Goodwin, and T. Fawcett, Transforming the UK Cold Market, DECADE - Domestic Equipment and Carbon Dioxide Emissions, Energy and Environment Programme, Environmental Change Unit, University of Oxford, Oxford, 1997.

[20] M.-A. Chavy-Macdonald, K. Oizumi, and K. Aoyama, A model framework for determining dynamic architecture goals in a System-of-Systems, In: A.M. Madni, et al. (eds.) Disciplinary Convergence in Systems Engineering Research, Springer Int. Publishing, 2018, doi: 10.1007/978-3-319-62217-0_17.

[21] E. Crawley, B. Cameron, and S. Selva, System Architecture: Strategy and Product Development for Complex Systems, 1st edition, Prentice Hall, Boston, 2015. 